\title{
MATHEMATICAL MODELING OF MAGNETO PULSATILE BLOOD FLOW THROUGH A POROUS MEDIUM WITH A HEAT SOURCE
}

\author{
B.K SHARMA* \\ Department of Mathematics \\ Birla Institute of Technology and Science \\ Pilani, Rajasthan, INDIA \\ E-mail: bhupen_1402@yahoo.co.in \\ M. SHARMA and R.K. GAUR \\ Department of Biotechnology, FASC \\ Mody University of Science and Technology \\ Lakshmangarh, Rajasthan, INDIA \\ A. MISHRA \\ Department of Aerospace Engineering \\ Indian Institute of Science \\ Bangalore, INDIA
}

\begin{abstract}
In the present study a mathematical model for the hydro-magnetic non-Newtonian blood flow in the non-Darcy porous medium with a heat source and Joule effect is proposed. A uniform magnetic field acts perpendicular to the porous surface. The governing non-linear partial differential equations have been solved numerically by applying the explicit finite difference Method (FDM). The effects of various parameters such as the Reynolds number, hydro-magnetic parameter, Forchheimer parameter, Darcian parameter, Prandtl number, Eckert number, heat source parameter, Schmidt number on the velocity, temperature and concentration have been examined with the help of graphs. The present study finds its applications in surgical operations, industrial material processing and various heat transfer operations.
\end{abstract}

Key words: heat source, blood flow, porous medium and MHD.

\section{Introduction}

Flow of blood through arteries is an important physiological problem, and is of high interest for biomedical researchers, physiologists, and clinicians. One of the important aspects of this problem that requires special attention is the deviation in the flow characteristics of blood when the artery is exposed to an externally applied magnetic field. This aspect has been the subject of vigorous research in recent years. Mathematical modeling of heat transfer in bio-fluid engineering was initiated in the late 1940s in a seminal paper by Pennes (1984) which laid the foundations for conduction heat transfer in tissue. The pulsatile fluid flow with heat transfer finds its applications in the fields such as mechanical and industrial thermal engineering systems. Baish (1990) studied heat transport by countercurrent blood vessels in the presence of an arbitrary pressure gradient. Consiglieri et al. (2003), Davalos et al. (2003) discussed the theoretical analysis of the heat convection coefficient in large vessels. An analytical study of heat transfer in finite tissue with two blood vessels and uniform Dirichlet boundary conditions was made by Shrivastava et al. (2005).

\footnotetext{
* To whom correspondence should be addressed
} 
These studies were all confined to Newtonian blood flow models. Although many Newtonian models considering Navier-Stokes equations are studied, the rheological nature of bio-fluids such as blood, plasma, bile etc. have also been recognized in a lot many studies. It is required to develop a non-Newtonian model that can present more accurate results in field of study of physiological fluids. Due to the pumping action of the heart in the human circulation system, a pulsatile feature of blood must also be considered. Skalak and Chien (1982) presented an investigation of the non-Newtonian flow of blood, considering erythrocytes as soft tissues. An excellent summary of a number of rheological models for blood was provided by Cokelet (1972).

In mid-1980's engineers became interested in the influence of magnetic fields on blood flows primarily with a view to utilize MHD (magneto-hydrodynamics) in controlling blood flow velocities in surgical procedures and also establishing the effects of magnetic fields on blood flows in astronauts, citizens living in the vicinity of EM (electro-magnetic) towers. The presence of iron oxides in the hemoglobin molecule has been shown (Takeuchi et al., 1995) to produce strong magnetic properties in blood. Under oxygenated conditions blood exhibits diamagnetic properties and under deoxygenated conditions it behaves as a paramagnetic fluid. Several authors have also reported on heat transfer in bio-magnetic fluid flows including (Tzirtzilakis and Tanoudis, 2003) for bio-magnetic convective heat transfer over a stretching surface and Louckopoulos and Tzirtzilakis (2004) for bio-magnetic flow and heat transfer in a parallel-plate system.

The presence of a porous medium during the flow presents a more physically realizable study. This approach can be modeled in the blood vessels and pulmonary systems due to the presence of fatty deposits and artery blockages. Generally, a Darcy model is most widely used for modeling the porous conditions but under higher pressure gradients and in highly porous regimes where inertial effects dominate viscous effects, the Darcian model flow is inadequate. Khaled and Vafai (2003) presented a rigorous review of heat and fluid dynamics applications in porous (biological) media. The effects of temporally-varying wall mass flux on hydromagnetic pulsatile Newtonian blood flow in a Darcian porous model of the cardiovascular system, using a regular perturbation technique were investigated by Ogulu and Amos (2007). Various studies using this model have been presented in the context of porous media heat transfer, as described by Pop and Ingham (2001). Significant works include those by Preziosi and Farina (2002) who studied mass exchange using an extended Darcy model, Vankan et al. (1997) who considered non-Darcy transport in blood perfused tissue and Sorek and Sideman (1986) who analyzed blood flow in cardiac vessels using a Darcy-Forchheimer model. Recently, Bhargava et al. (2007) used the Darcy-Forchheimer model to analyze pulsating magnetohydrodynamic blood flow and species diffusion in a porous medium channel. Joule dissipation acts as a volumetric heat source and finds its practical interest due to large industrial applications. Recently, Sharma $e t$ al. (2013) studied heat and mass transfer in a magnetic bio fluid flow through a non-Darcian porous medium with Joule effect

In the above mentioned studies the effects of heat sources/sinks have not been considered. A recent pilot study, however, indicates that the moisture content of the skin may also alter the response of vascular endothelial cells to local heat (KcLellan et al., 2009). In these studies, if the skin was kept dry during warming, the skin blood flow response was significantly smaller than if moist heat was used as a heating source (KcLellan et al., 2009). There is some evidence in the literature to support this concept. Petrofsky et al. (2009) noted that the increased blood flow response observed in the skin could have been due to the moisture in the heat source or the faster rate of rise in skin temperature or both. Kandasamy et al. (2005) discussed heat and mass transfer effect along a wedge with a heat source and concentration in the presence of suction/injection taking into account the chemical reaction of first order. Sharma et al. $(2007 ; 2007)$ studied MHD free convective flow of a viscous fluid past an infinite vertical porous plate with heat source/sink effect. Unsteady MHD convective Heat and Mass transfer past a semi-infinite vertical permeable moving plate with heat absorption was discussed by Chambkha (2004). Sharma et al. $(2007 ; 2008 ; 2011)$ discussed the radiation effect on a free convective flow along a uniform moving porous vertical plate in the presence of a heat source/sink and transverse magnetic field. Recently, Soret and Dufour effects on an unsteady MHD mixed convective flow past an infinite vertical plate with Ohmic dissipation and heat source were studied by Sharma et al. (2014). Hence, the main objective of the present investigation is to study a 
biological fluid flow with heat and mass transfer considering its pulsatile hydro-magnetic rheological nature under the presence of viscous dissipation, Joule heating and a finite heat source through a Darcian porous medium.

\section{Mathematical formulation}

Consider an unsteady two-dimensional rheological bio-fluid flow between the narrow channel in the presence of a porous medium with viscous dissipation, Joule dissipation and heat source effects. The plates are kept at a distance of $2 \mathrm{H}$ with wall transpiration effects. The pulsatile nature of a bio-fluid like blood is considered. Blood is pumped through the human cardiovascular system by the heart. Here, the source parameter effect has been considered by taking real pistons in action. The transverse magnetic field $\left(B_{0}\right)$ is applied considering the electrical nature of the bio-fluid. The porous medium here is of non-Darcian nature and the wall transpiration is present as injection at $(-\mathrm{H})$ and suction at $(\mathrm{H})$. The Joule dissipation and heat source/sink have also been included in the energy equation. The fluid here is non-Newtonian in nature; therefore the Nakamura-Sawada model especially used for such cases has been employed. The mass conservation for the species is considered by taking its concentration $C_{1}$ at the lower plate $(-\mathrm{H})$ and $C_{2}$ at the upper plate $(\mathrm{H})$. Thus, under these assumptions, the problem is governed by the following system of equations:

\section{Linear Momentum Equation}

$$
\frac{\partial u}{\partial \tau}+V_{0} \frac{\partial u}{\partial y}+\frac{l}{\rho} \frac{\partial P}{\partial x}=v_{B}\left(1+\frac{l}{\beta}\right) \frac{\partial^{2} u}{\partial y^{2}}-\frac{\sigma B_{0}^{2}}{\rho} u-\frac{v_{B}}{k_{p}} u-b u^{2} .
$$

\section{Energy Equation}

$$
\frac{\partial T}{\partial \tau}+V_{0} \frac{\partial T}{\partial y}=\alpha \frac{\partial^{2} T}{\partial y^{2}}+\frac{v_{B}}{c_{p}}\left(\frac{\partial u}{\partial y}\right)^{2}+s^{*}\left(T-T_{m}\right)+\frac{\sigma B_{0}^{2}}{\rho c_{p}} u^{2}
$$

\section{Concentration Equation}

$$
\frac{\partial C^{\prime}}{\partial \tau}+V_{0} \frac{\partial C^{\prime}}{\partial y}=D \frac{\partial^{2} C^{\prime}}{\partial y^{2}}
$$

The corresponding boundary conditions are

$$
\begin{aligned}
& y=-H: \quad u=0 ; \quad T=T_{1} ; \quad C^{\prime}=C_{l}, \\
& y=H: \quad u=0 ; \quad T=T_{2} ; \quad C^{\prime}=C_{2}
\end{aligned}
$$

where $\mu_{B}$ is Newtonian dynamic viscosity, $V_{o}$ is the wall transpiration velocity ( $V=V_{o}$ at the lower plate and $V=-V_{o}$ at the upper plate), $\beta$ denotes the upper limit of the apparent viscosity coefficient, where $u$ is the $x$ direction (longitudinal velocity), $P$ is the hydrodynamic pressure, $k_{p}$ is the hydraulic conductivity (permeability) of the porous material, $\rho$ is the density of the fluid, $b$ is a Forchheimer coefficient related to the porous medium geometry, $\tau$ is the dimensional time, $\sigma$ is the electrical conductivity of the bio-fluid, $B_{0}$ is the transverse magnetic field strength, $\alpha$ is the thermal diffusivity, $c_{p}$ is the specific heat capacity of the biofluid, $s^{*}$ is the source parameter, $T$ is the bio-fluid temperature, $C$ is the species concentration, $D$ is the mass diffusivity of the species, $\partial P / \partial x$ denotes the longitudinal pressure gradient. 
Introducing the following non-dimensional parameters

$$
\begin{aligned}
& U=\frac{u}{V_{0}}, \quad X=\frac{x}{H}, \quad Y=\frac{y}{H}, \quad t=\frac{V_{0}}{H} \tau, \quad P^{*}=\frac{P}{\rho V_{0}}, \quad \Phi=\frac{T-T_{m}}{T_{2}-T_{m}}, \quad \operatorname{Re}=\frac{H V_{0}}{v_{B}}, \\
& N m=\frac{\sigma B_{0} H}{\rho V_{0}}, \quad \lambda=\frac{k_{p} V_{0}}{v_{B} H}, \quad \mathrm{Nf}=H b, \quad \operatorname{Pr}=\frac{v_{B}}{\alpha}, \quad \mathrm{Ec}=\frac{V_{0}^{2}}{c_{p}\left(T_{2}-T_{m}\right)}, \quad \mathrm{Sc}=\frac{H V_{0}}{D}, \\
& C=\frac{C^{\prime}-C m}{C_{2}-C_{m}}, \quad S=\frac{s^{*}}{V_{0}} .
\end{aligned}
$$

Equations (2.1) to (2.3) are reduced to the following non-dimensional form:

\section{Momentum Equation}

$$
\frac{\partial U}{\partial t}+\frac{\partial U}{\partial Y}+\frac{\partial P}{\partial X}=\frac{1}{\operatorname{Re}}\left(1+\frac{1}{\beta}\right) \frac{\partial^{2} U}{\partial Y^{2}}-N m U-\frac{1}{\lambda} U-\mathrm{Nf} U^{2} .
$$

\section{Energy Equation}

$$
\frac{\partial \theta}{\partial t}+\frac{\partial \theta}{\partial Y}=\frac{1}{\operatorname{Pr} \operatorname{Re}} \frac{\partial^{2} \theta}{\partial Y^{2}}+\frac{\operatorname{Ec}}{\operatorname{Re}}\left(\frac{\partial U}{\partial Y}\right)^{2}+S \theta+\operatorname{Ec} N m U^{2}
$$

\section{Concentration Equation}

$$
\frac{\partial C}{\partial t}+\frac{\partial C}{\partial Y}=\frac{1}{\mathrm{Sc}} \frac{\partial^{2} C}{\partial Y^{2}}
$$

The transformed boundary conditions become

$$
\begin{aligned}
& Y=-1: \quad U=0 ; \quad \Theta=-1 ; \quad C=-1, \\
& Y=1: \quad U=0 ; \quad \Theta=1 ; \quad C=1
\end{aligned}
$$

where $X$ and $Y$ are dimensionless coordinates parallel and transverse to the bio-fluid flow, respectively, $U$ is the transformed velocity component in the $X$-direction, $P^{*}$ is the transformed hydrodynamic pressure (* dropped for convenience in analysis), $t$ is the dimensionless time, $\theta$ is the dimensionless temperature, $\operatorname{Re}$ is a transpiration Reynolds number, $\mathrm{Nm}$ is the hydromagnetic parameter, $\lambda$ is a Darcian (permeability) parameter, $\mathrm{Nf}$ is the Forchheimer (quadratic porous drag) parameter, $T m=\left(T_{1}+T_{2}\right) / 2$ is the characteristic temperature, $\mathrm{Cm}=\left(C_{1}+C_{2}\right) / 2$ is the characteristic concentration, $\mathrm{Pr}$ is the Prandtl number, Ec is the Eckert Number, Sc is the Schmidt number and $S$ is the dimensionless source parameter.

As the present problem of fluid flow is pulsatile in nature, therefore the pressure gradient component is decomposed into a steady component and an oscillatory component as below

$$
-\frac{\partial P}{\partial X}=\left(\frac{\partial P}{\partial X}\right)_{S}+\left(\frac{\partial P}{\partial X}\right)_{o} e^{i \omega t}
$$


First, to solve the above Eqs (2.4)-(2.5) coupled equations, the pressure term is redefined as

$$
-\frac{\partial P}{\partial X}=P_{s}+P_{0} \cos \left(w^{*} t\right)
$$

where $P s=$ the static pressure component, $P_{0}=$ the oscillatory pressure component.

\section{Method of solution}

The above nonlinear dimensionless partial differential equations with boundary conditions have been solved numerically by applying the Explicit Finite Difference Technique. The finite difference equations corresponding to Eqs (2.4) to (2.6) are as follows

$$
\begin{aligned}
& \frac{U_{i}{ }^{j+1}-U_{i}{ }^{j-1}}{2 \Delta t}+\frac{U_{i+1}{ }^{j}-U_{i-1}^{j}}{2 \Delta Y}+\left(P_{s}+P_{0} \cos \omega t\right)= \\
& =\frac{1}{\operatorname{Re}}\left(1+\frac{1}{\beta}\right) \frac{U_{i+1}^{j}-2 U_{i}^{j}+U_{i-1}^{j}}{\Delta Y^{2}}-\left(N m+\frac{1}{\lambda} M\right) U_{i}{ }^{j}-\mathrm{Nf}\left(U_{i}^{j}\right)^{2}, \\
& \frac{\theta_{i}^{j+1}-\theta_{i}^{j-1}}{2 \Delta t}+\frac{\theta_{i+1}^{j}-\theta_{i-1}{ }^{j}}{2 \Delta Y}=\frac{1}{\operatorname{Pr} \operatorname{Re}} \frac{\theta^{j}{ }_{i+1}-2 \theta^{j}{ }_{i}+\theta^{j}{ }_{i-1}}{\Delta Y^{2}}+ \\
& +S \theta_{i}{ }^{j}+\frac{\operatorname{Ec}}{\operatorname{Re}}\left(\frac{U_{i+1}{ }^{j}-u_{i-1}{ }^{j}}{2 \Delta Y}\right)^{2}+N m \operatorname{Ec}\left(U_{i}{ }^{j}\right)^{2}, \\
& \frac{C_{i}{ }^{j+1}-C_{i}{ }^{j-1}}{2 \Delta t}+\frac{C_{i+1}{ }^{j}-C_{i-1}{ }^{j}}{2 \Delta Y}=\frac{1}{\mathrm{Sc}} \frac{C^{j}{ }_{i+1}-2 C^{j}{ }_{i}+C^{j}{ }_{i-1}}{\Delta Y^{2}} .
\end{aligned}
$$

To obtain the difference equations, the region of the flow is divided into a gird or mesh of lines parallel to $Y$ and $t$ axes. Solutions of difference equations are obtained at the intersection of these mesh lines called nodes. The finite-difference equations at every internal nodal point on a particular $n$-level constitute a tri-diagonal system of equations. These equations are solved by using the Thomas algorithm (Hoffman, 1992).

In order to prove the convergence of the finite difference scheme, the computation is carried out for slightly changed values of $\Delta Y$ and, $\Delta t$. Negligible changes are observed in the values of $u, T$, and $C$ and also after each cycle of iteration the convergence checking is performed i.e., $\left|f^{n+1}-f^{n}\right|<10^{-8}$ is satisfied at all points. Thus, due to computational cost and accuracy the above mesh size was considered optimal.

\section{Results and discussion}

The hydro-magnetic non-Newtonian bio-fluid flow in the non-Darcy porous medium with a heat source and Joule effect has been discussed in preceding sections. In order to get a physical insight into the problem, the numerical calculations for the distribution of the velocity, temperature and concentration for various dimensionless parameters were made. The bio-fluid is blood and the values of various parameters used are taken as in Sharma et al. (2013). In order to access the accuracy of our results, comparisons have 
been made with Rawat et al. (2009) and results are shown in Tab.1. An excellent agreement can be observed between both studies for the dimensionless velocity profile $(U)$ with the transverse coordinate $(Y)$.

Table 1. Comparison of present study and Rawat et al. (2009).

\begin{tabular}{|l|l|l|}
\hline \multicolumn{2}{|l|}{$\operatorname{Re}=0.5, \beta=4, \omega=8, \operatorname{Ps}=10, P o=7, \lambda=5, \mathrm{Nf}=0.002, N m=0.3, \mathrm{Ec}=6^{*} 10^{-9}, \operatorname{Pr}=21$} \\
\hline & $U($ at $t=0.5)$ & Present study \\
\hline$Y$ & Rawat et al. $(2009)$ & 0 \\
\hline-1 & 0 & 0.477452 \\
\hline-0.65854 & 0.477402 & 0.792369 \\
\hline-0.31707 & 0.792337 & 0.866126 \\
\hline 0.31707 & 0.866090 & 0.572932 \\
\hline 0.658537 & 0.572876 & 0 \\
\hline 1 & 0 & \\
\hline
\end{tabular}

Figure 1 depicts the velocity profile for different values of the Reynolds number (Re) at $t=0.5$. As the Reynolds number involves transpiration velocity, hence an increase in Re results in an increase of velocity $(U)$ values and generally the peaks are shifted towards the right. We note that the Reynolds number of unity implies a creeping flow regime, i.e., where the inertial core encountered in higher velocity porous flows has not developed, as discussed by Dybbs and Edwards (1984). Figure 2 shows the effects of the nonNewtonian parameter $(\beta)$ on the velocity. Lower values of $\beta$ imply higher values of viscosity and thus the lower values of velocity. As $\beta$ increases the velocity increases and so the peak will be maximum as $\beta$ approaches infinity. Figure 3 shows the influence of the Darcian parameter $(\lambda)$ on the velocity profiles. Higher values of $\lambda$ imply lower fatty deposits and blockages in the fluid flow channel, resulting in lower resistances and higher velocities. The effects of the hydro-magnetic parameter $(\mathrm{Nm})$ are depicted in Fig.4. As the values of $\mathrm{Nm}$ increase, the velocity parabola is lowered. This is due to the retarding forces (Lorentz forces) generated by the magnetic field as bio-fluid's electrical properties have been already confirmed. As such magnetic fields are extremely useful in regulating blood flows in practical applications.

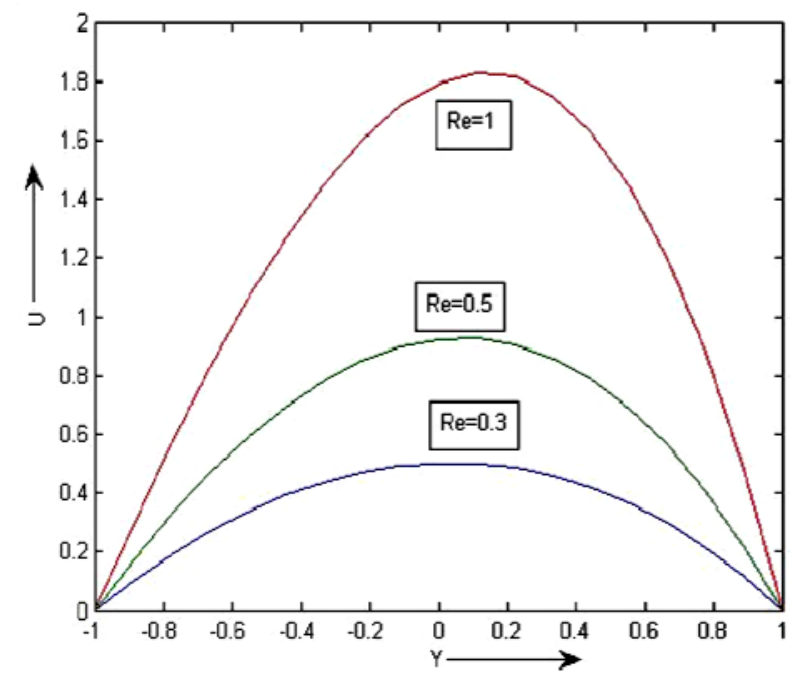

Fig.1. $U$ versus $Y$ for various transpiration Reynolds numbers $(\mathrm{Re})$ at $t=0.5$.

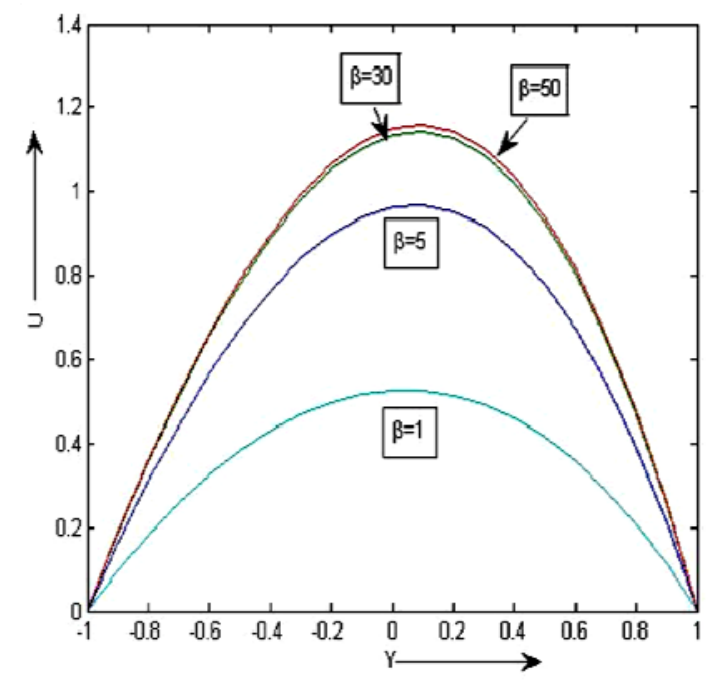

Fig.2. $U$ versus $Y$ for various non- Newtonian parameter values $(\beta)$ at $t=0.5$. 


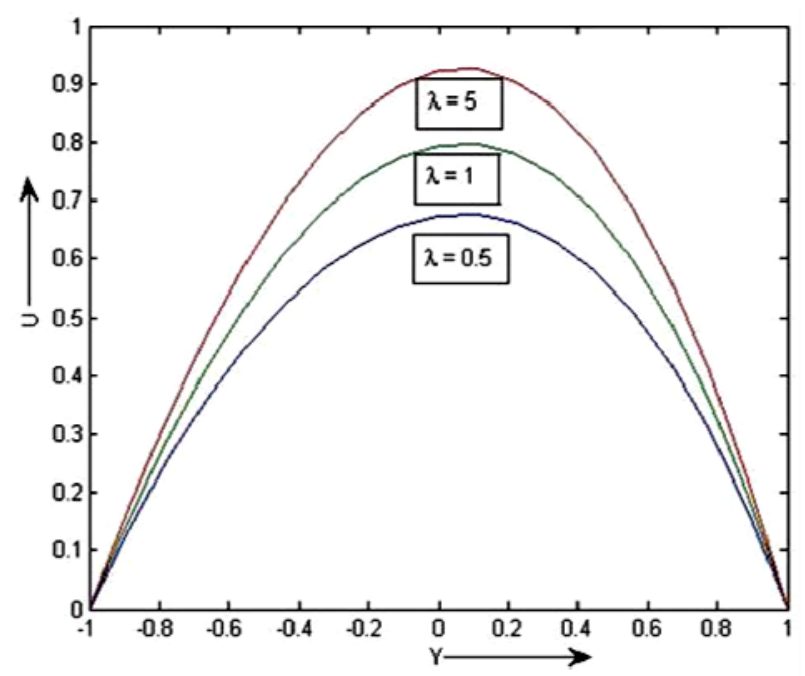

Fig.3. $U$ versus $Y$ for various Darcian parameter values $(\lambda)$ at $t=0.5$.

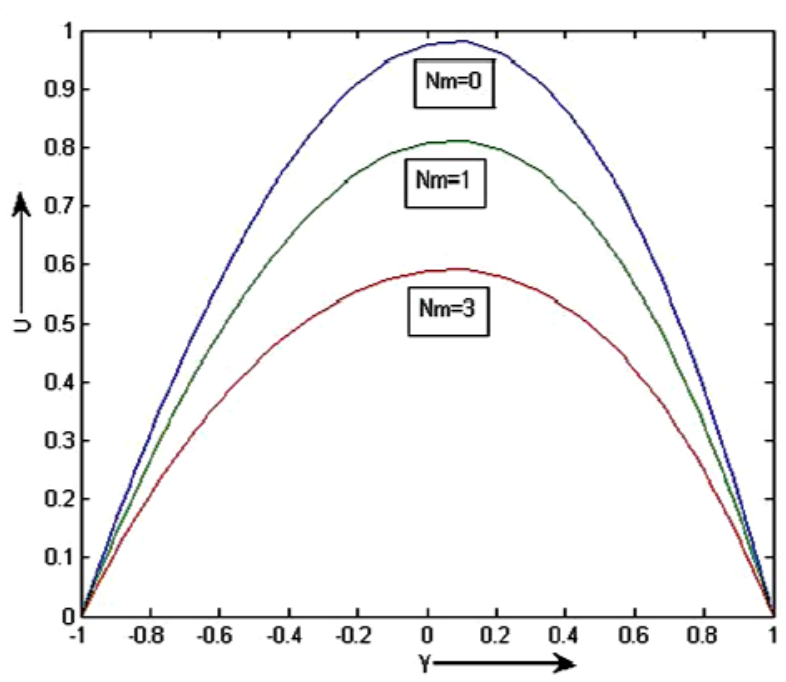

Fig.4. $U$ versus $Y$ for various hydromagnetic parameter values $(\mathrm{Nm})$ at $t=0.5$.

In Fig.5, the influence of the hydro-magnetic parameter $(\mathrm{Nm})$ at two Ec values on the temperature is depicted. As earlier known $\mathrm{Nm}$ has an inhibiting effect on velocity and thus on temperature due to the Joule dissipation term in Eq.(2.4). The Joule dissipation term acts as a volumetric heat source, which becomes more important at higher Ec values. In Fig.5a, it is noticed that as $\mathrm{Nm}$ increases the temperature peaks decrease and in Fig.5b higher Ec value results in more heat generation and larger temperature values and the oscillations are increased due to the oscillatory nature of the velocity profile. The effects of the Prandtl number (Pr) for two different values of Ec on temperature distribution are shown in Fig.6. Pr represents the ratio of momentum diffusivity to thermal diffusivity. Larger Pr fluids $(\operatorname{Pr}>1)$ will diffuse momentum faster than heat. The numerical results show that the temperature decreases with an increase in the Prandtl number. This is due to the fact that a fluid with a high Prandtl number has a relatively low thermal conductivity which results in the reduction of the thermal boundary layer thickness.
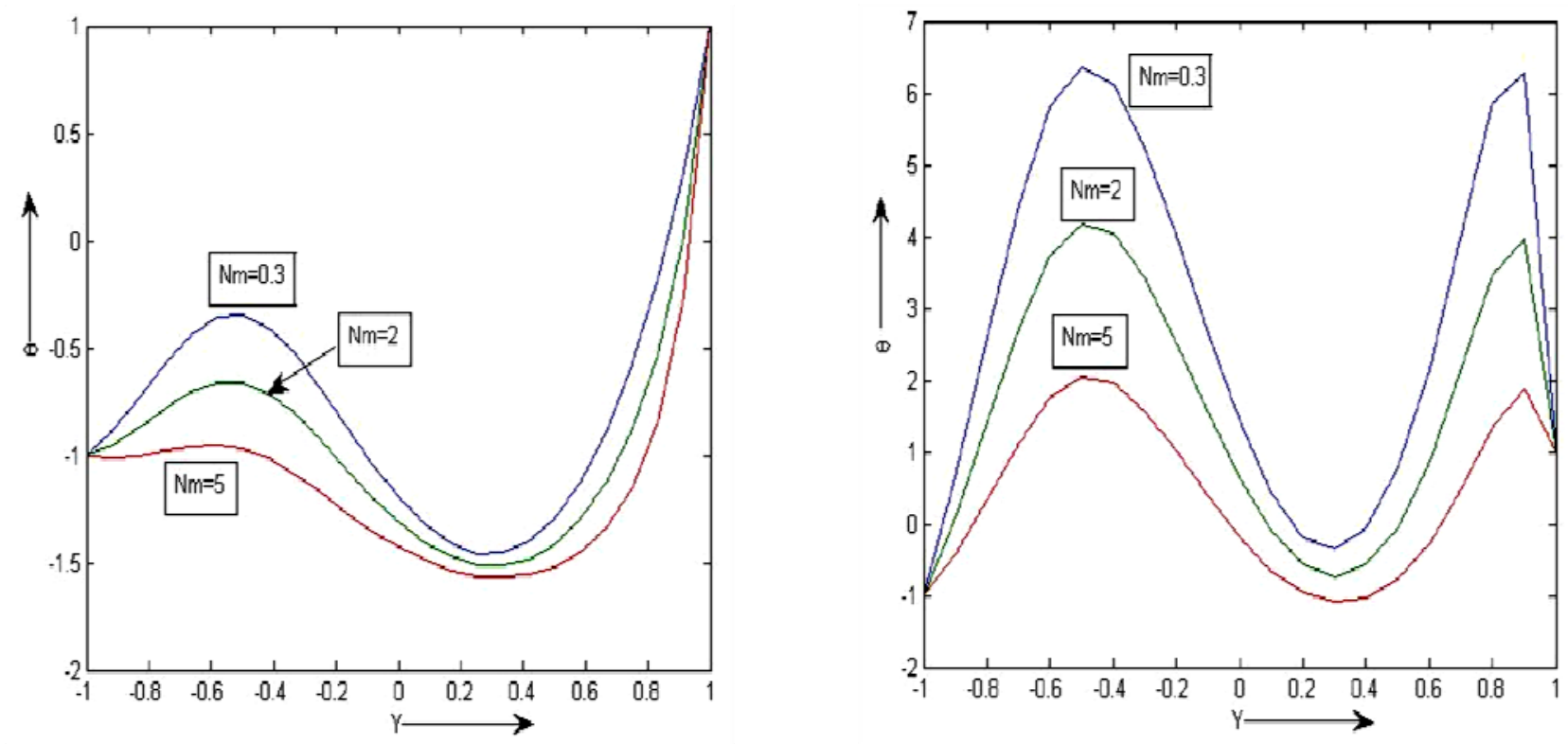

Fig.5. $\theta$ versus $Y$ for various hydromagnetic parameter values $(\mathrm{Nm})$ at $t=0.5$ for two Eckert numbers (a) $\mathrm{Ec}=0.0006$ (b) $\mathrm{Ec}=0.006$. 

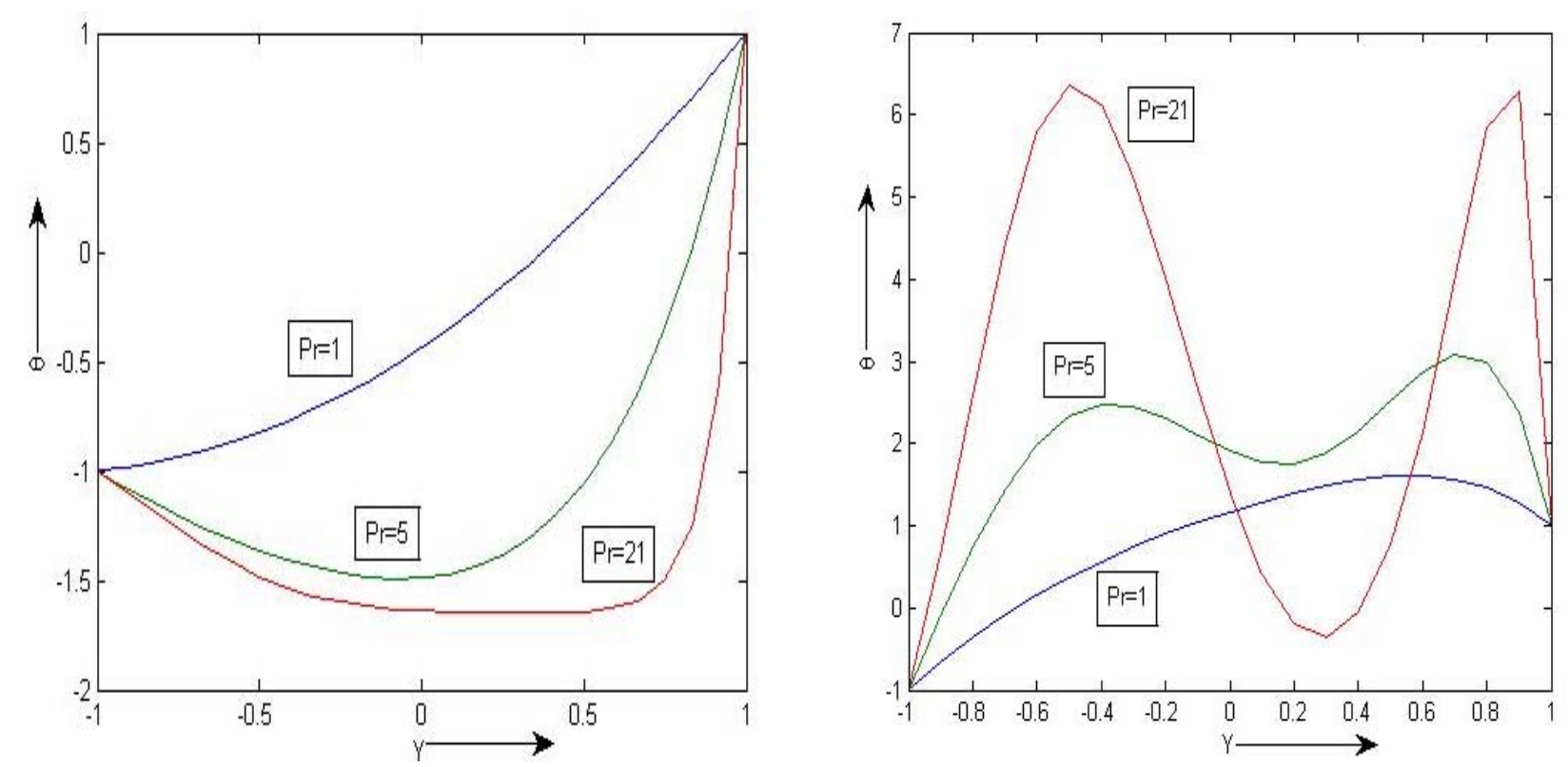

Fig.6. $\theta$ versus $Y$ for different values of $\operatorname{Pr}$ at $t=0.5$ for two Eckert numbers (a) $E c=6 \times 10^{-9}$ (b) $E c=0.006$.

In Fig.7 and Fig.8, the influence of the heat source parameter $(S)$ taking both positive and negative values at two Ec values is illustrated. In Fig. $7 \mathrm{a}$ and $7 \mathrm{~b}$, the heat sink parameter $(S<0)$ effects are shown. It can be observed that effects of $S$ are more pronounced at its higher negative values. More negative values result in higher temperatures but at higher Eckert number, larger oscillations result in a reversal of sink parameter effects and higher temperatures are observed at higher Ec and lower $S$ value. Figures $8 \mathrm{a}$ and $8 \mathrm{~b}$ depict the effect of the heat source parameter $(S>0)$. For Ec $=0.0006$, higher $S$ values imply lower temperature due to the cooling between the two plates, but the upper plate boundary condition results in the value of $\Theta=1$ at the upper plate. Similarly, higher Ec $(=0.006)$ results in more oscillations and higher temperatures.
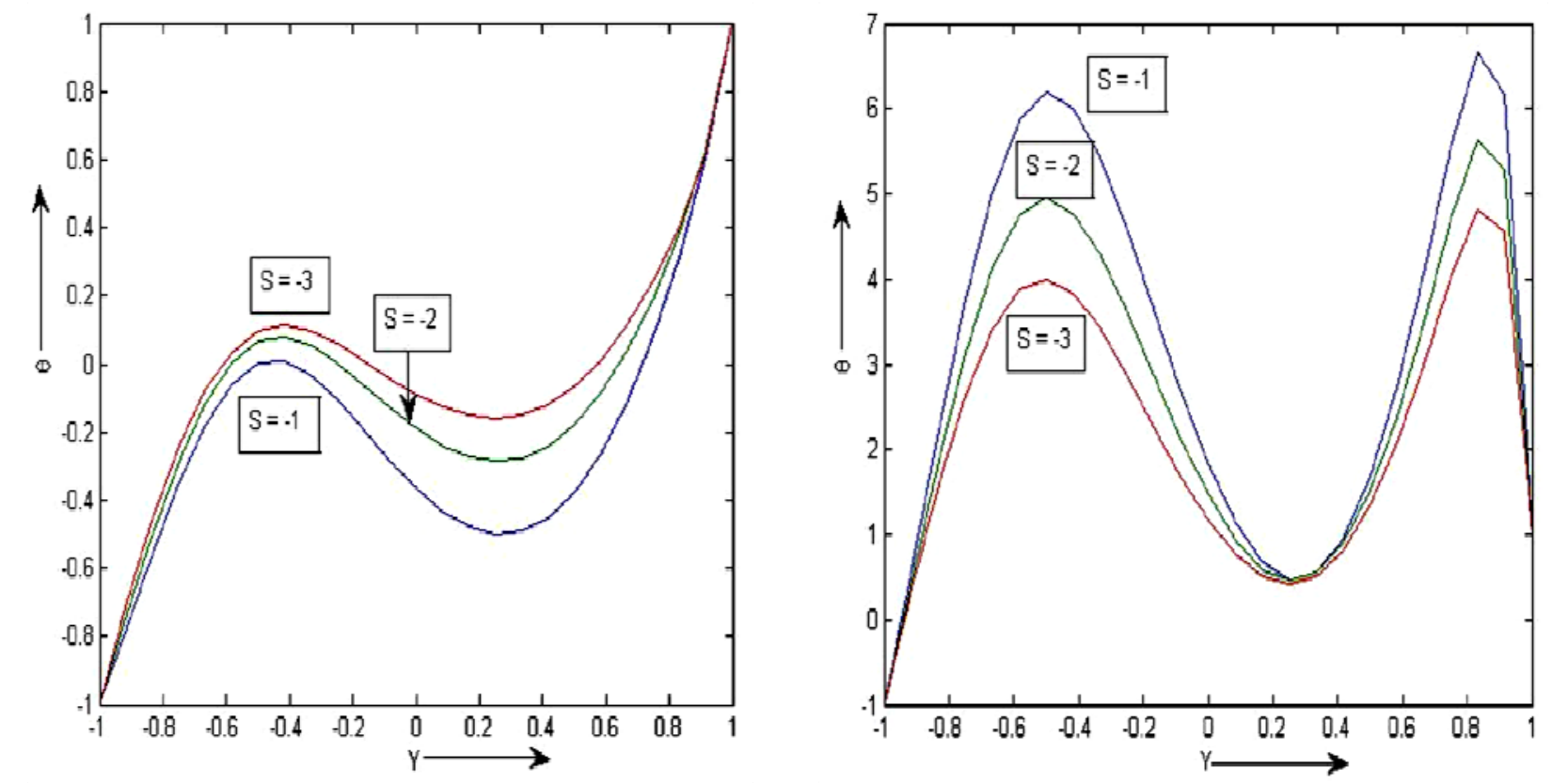

Fig.7. $\theta$ versus $Y$ for various sink parameter values(S) at $t=0.5$ for two Eckert numbers (a) Ec $=0.0006$ (b) $\mathrm{Ec}=0.006$. 

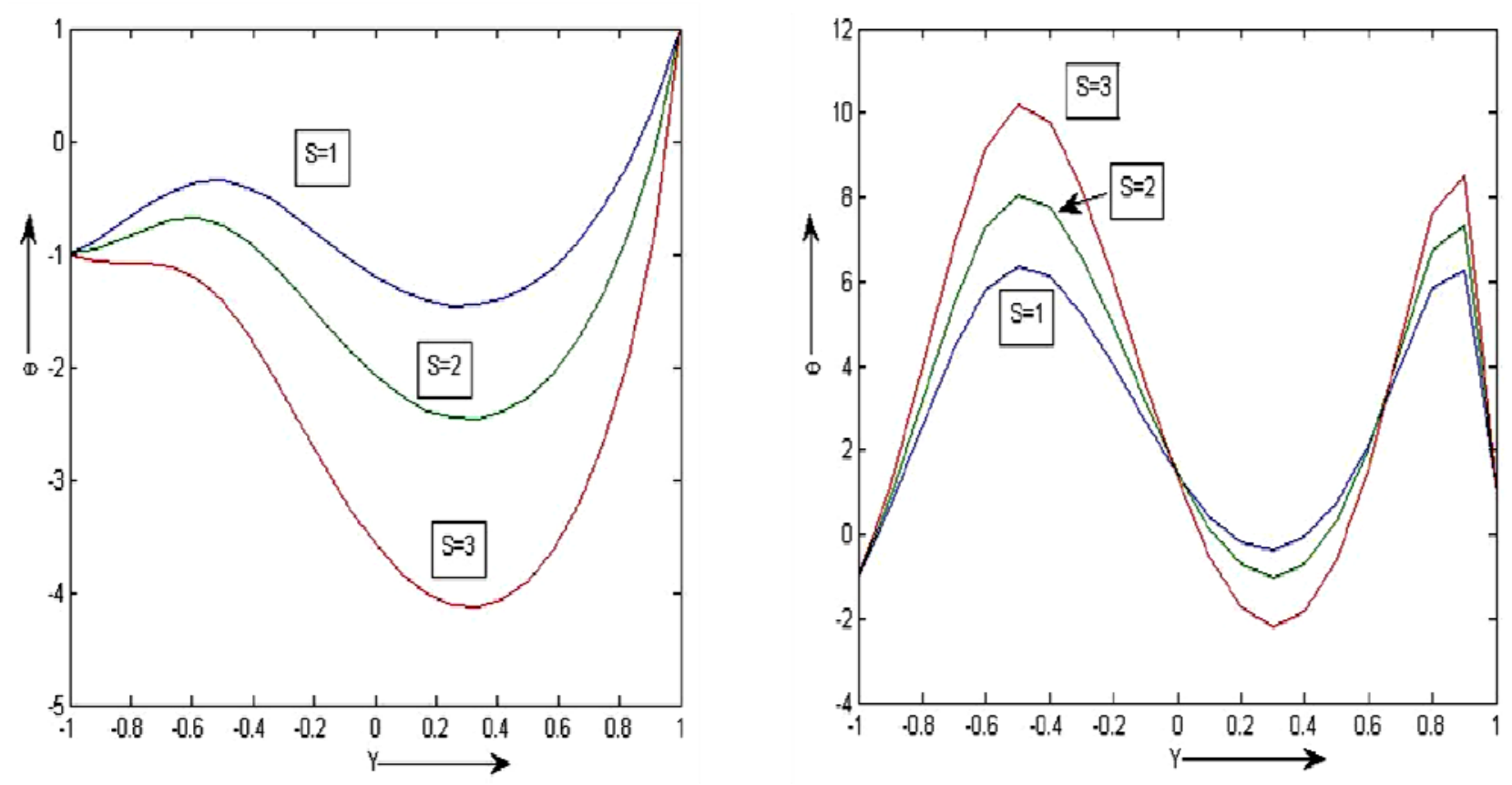

Fig.8. $\theta$ versus $Y$ for various source parameter values $(S)$ at $t=0.5$ for two Eckert numbers $\mathrm{Ec}=0.0006$ (b) $\mathrm{Ec}=0.006$.

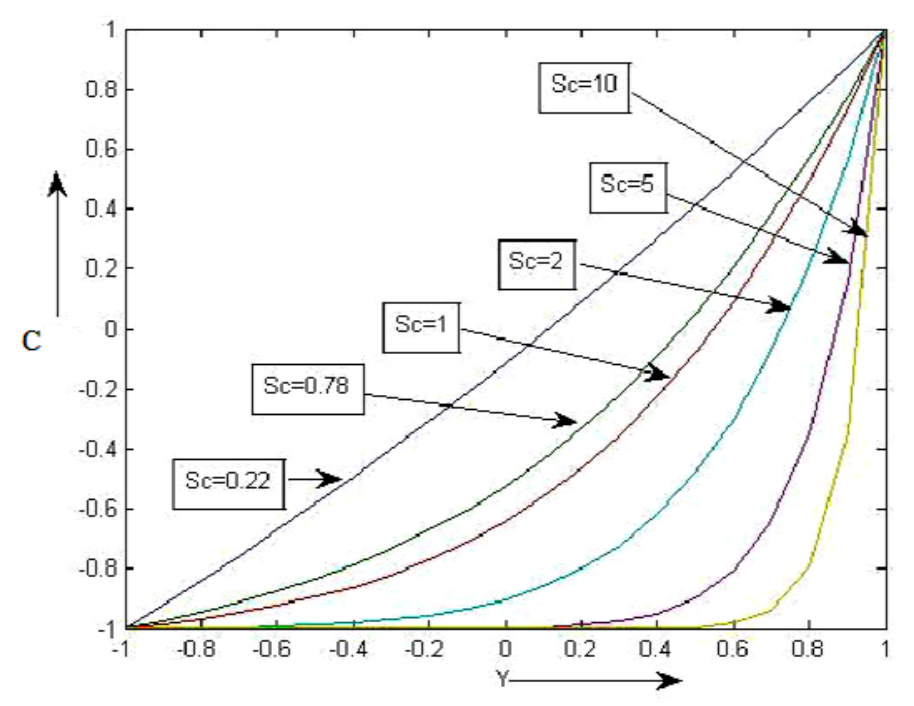

Fig.9. Concentration versus $Y$ for various Schmidt numbers (Sc) at $t=0.5$.

In Fig.9, the effect of the Schmidt number (Sc) over the species concentration in the channel has been shown for its various values. The Schmidt number defines the relative effectiveness of momentum and mass transport by diffusion in the velocity and concentration fields. Lower Sc values physically represent for example in industrial applications, hydrogen gas as the species diffusing $(\mathrm{Sc}=0.22)$, ammonia $(\mathrm{Sc}=0.78)$, methanol diffusing in air $(\mathrm{Sc}=1.0)$ and ethylbenzene in air $(\mathrm{Sc}=2.0)$. Sc physically represent the relativeness of momentum and mass diffusion. A comparison of curves in the figure shows a decrease in the concentration $(C)$ with an increase in the Schmidt number $(\mathrm{Sc})$. Physically, the increase of Sc means a decrease of the molecular diffusivity $(D)$, that results in a decrease of the concentration boundary layer. Hence, the concentration of the species is higher for small values of Sc and lower for larger values of Sc. 


\section{Nomenclature}

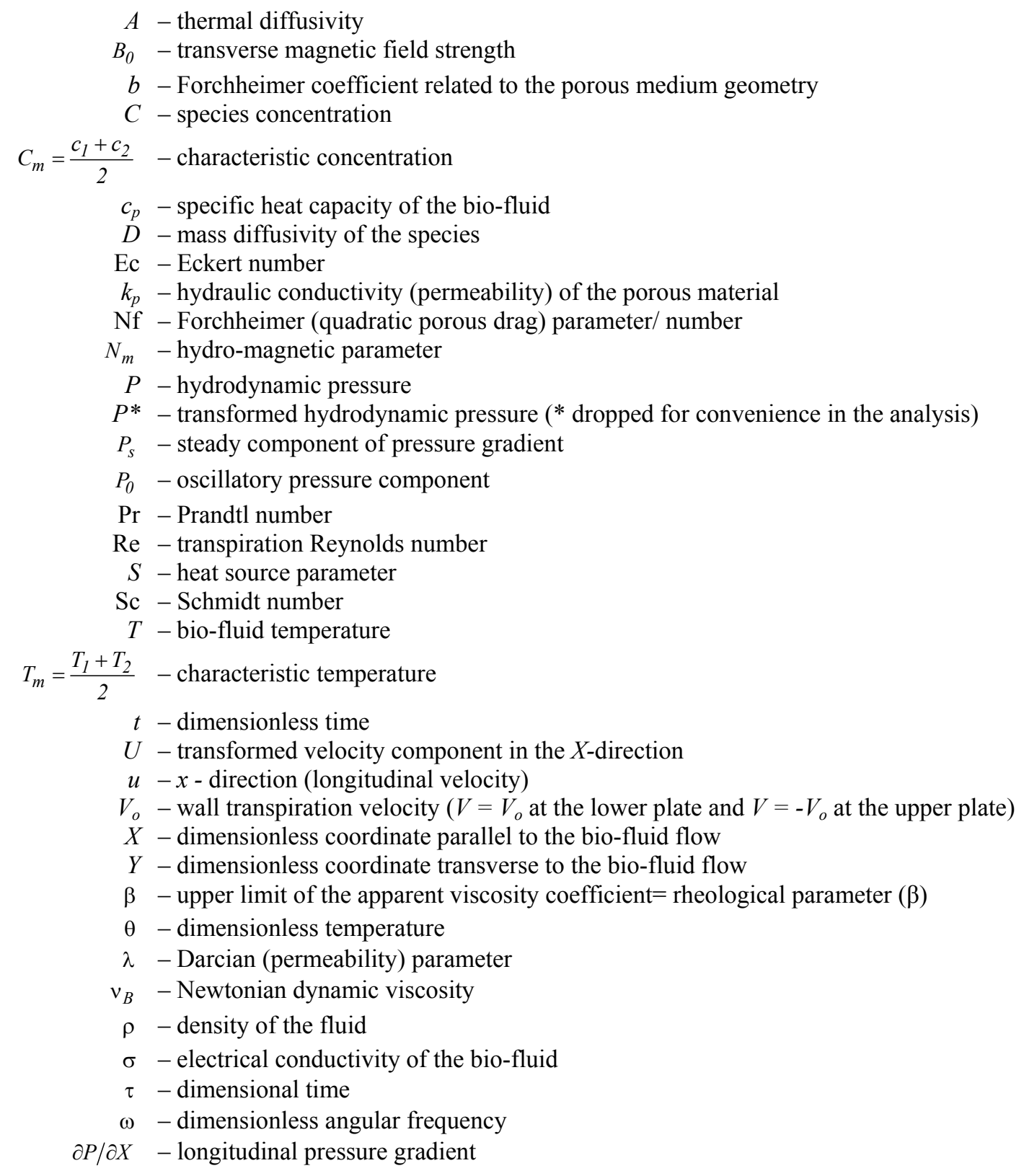

\section{References}

Baish J.W. (1990): Heat transport by countercurrent blood vessels in the presence of an arbitrary pressure gradient. ASME J Biomech. Eng., 112:207.

Bhargava R., Rawat S., Takhar H.S. and Bég O.A. (2007): Pulsatile magneto-biofluid flow and mass transfer in a nonDarcian porous medium channel. - Meccanica, vol.42, pp.247-262.

Chamkha Ali J. (2004): Unsteady MHD convective heat and mass transfer past a semi-infinite vertical permeable moving plate with heat absorption. - Int. J. Engg. Sci., vol.42, pp.217-230. 
Cokelet G.R. (1972): The rheology of human blood. In: Fung YC (ed) Biomechanics-its foundations and objectives. New York: Prentice Hall.

Consiglieri L., Santos I. and Haemmerich D (2003): Theoretical analysis of the heat convection coefficient in large vessels and the significance for thermal ablative therapies. - Phys. Med. Biol., vol.48, pp.4125-4134.

Davalos R.V., Rubinsky B. and Mir L.M. (2003): Theoretical analysis of the thermal effects during in vivo tissue electroporation. - Bioelectrochem J., vol.61, pp.99-107.

Dybbs A. and Edwards R.V. (1984): A new look at porous media fluid mechanics: Darcy to Turbulent. In: Bear J., Corapcioglu M.Y. (eds) Fundamentals of Transport Phenomena in Porous Media NATO ASI series E: Applied Sciences, vol.82, pp.199-256.

Hoffman J.D. (1992): Numerical Methods for Engineers and Scientists. - New York: McGraw-Hill.

Kandasamy R., Periasamy K. and Prashu Sivagnana K.K. (2005): Effects of chemical reaction, heat and mass transfer along wedge with heat source and concentration in the presence of suction or injection. - Int. J. Heat Mass Transfer, vol.48, pp.1388-1394.

Khaled A.R.A. and Vafai K. (2003): The role of porous media in modeling flow and heat transfer in biological tissues. - Int. J. Heat and Mass Transfer, vol.46, pp.4989-5003.

Louckopoulos V.C. and Tzirtzilakis E.E. (2004): Biomagnetic channel flow in spatially varying magnetic field. - Int. J. Eng. Sci., vol.42, pp.571-590.

McLellan K., Petrofsky J.S., Bains G., Zimmerman G., Prowse M. and Lee S. (2009): The effects of skin moisture and subcutaneous fat thickness on the ability of the skin to dissipate heat in young and old subjects, with and without diabetes, at three environmental room temperatures. - Med. Eng. Phys., vol.31, No.2, pp.165-172. doi:10.1016/ j.medengphy.2008.08.004

Ogulu A. and Amos E. (2007): Modeling pulsatile blood flow within a homogeneous porous bed in the presence of a uniform magnetic field and time-dependent suction. - Int. Comm. Heat Mass Transfer, vol.34, pp.989-995.

Pennes H.H. (1948): Analysis of tissue and arterial blood temperatures in the resting human forearm. - J. Appl. Physiol., vol.1, No.22, pp.93-122.

Petrofsky J.S., Bains G., Raju C., Lohman E., Berk L., Prowse M., Gunda S., Madani P. and Batt J. (2009): The effect of the moisture content of a local heat source on the blood flow response of the skin. - Archives of Dermatological Research, vol.301, No.8, pp.581-585.

Pop I. and Ingham D.B. (2001): Convective Heat Transfer: Mathematical and Numerical Modelling of Viscous Fluids and Porous Media, Pergamon, Oxford.

Preziosi L. and Farina A. (2002): On Darcy's law for growing porous media. - Int. J. Non-Linear Mech., vol.37, pp.485-491.

Rawat S., Bhargava R., Anwar Bég O., Bhargava P. and Hughes Ben R. (2009): Pulsatile dissipative magneto-biorheological fluid flow and heat transfer in a non-Darcy porous medium channel: finite element modeling. Emirates Journal for Engineering Research, vol.14, No.2, pp.77-90.

Sharma B.K., Agarwal M. and Chaudhary R.C. (2007): MHD fluctuating free convective flow with radiation embedded in porous medium having variable permeability and heat source/sink. - Journal of Technical Physics, vol.47, No.1, pp.47-58.

Sharma B.K., Agarwal M. and Chaudhary R.C. (2007): Effects of injection/suction on three-dimensional Couette flow with heat source/sink. - Ind. J. of Theoretical Physics, vol.55, No.1, pp.27-37.

Sharma B.K., Chaudhary R.C. and Agarwal M. (2008): Radiation effect on steady free convective flow along a uniform moving porous vertical plate in presence of heat source/sink and transverse magnetic field. - Bull. Cal. Math. Soc., vol.100, pp.529-538.

Sharma B.K., Gupta S., Krishna V.V. and Bhargavi R.J. (2014): Soret and Dufour effects on an unsteady MHD mixed convective flow past an infinite vertical plate with Ohmic dissipation and heat source. - Afrika Matematika, vol.25, pp.799-821, DOI 10.1007/s13370-013-0154-6. 
Sharma B.K., Jha A.K. and Chaudhary R.C. (2007): Hall effect on MHD free convective flow of a viscous fluid past an infinite vertical porous plate with Heat source/sink effect. - Romania Journal of Physics, vol.52, No.5-6, pp.487504.

Sharma B.K., Mishra A. and Gupta S. (2013): Heat and mass transfer in magneto-biofluid flow through a non-Darcian porous medium with Joule effect. - J. Eng. Phys. and Thermo Phys., vol.86, No.4, pp.716-725.

Sharma B.K., Sharma P.K. and Chand T. (2011): Effect of radiation on temperature distribution in three-dimensional Couette flow with heat source/sink. - International Journal of Applied Mechanics and Engineering, vol.16, No.2, pp.531-542.

Shrivastava D., McKay B. and Romer R.B. (2005): An analytical study of heat transfer in finite tissue with two blood vessels and uniform Dirichlet boundary conditions. - ASME J. Heat Transfer, vol.127, No.2, 179-188.

Skalak R. and Chien S. (1982): Rheology of blood cells as soft tissues. - Biorheology, vol.19, pp.453-461.

Sorek S. and Sideman S. (1986): A porous medium approach for modelling heart mechanics, B l-D case. - Math. Biosci., vol.81, pp.14-32.

Takeuchi T., Mizuno T., Higashi T., Yamagishi A. and Date M. (1995): Orientation of red blood cells in high magnetic field. - J. Magn. Magn. Mater., vol.140, pp.1462-1463.

Tzirtzilakis E.E. and Tanoudis G.B. (2003): Numerical study of biomagnetic fluid flow over a stretching sheet with heat transfer. - Int. J. Numer. Methods Heat Fluid Flow, vol.13, No.7, pp.830-848.

Vankan W.J., Huyghe J.M., Janssen J.D., Huson A., Hacking W.J.G. and Schrenner W. (1997): Finite element analysis of blood flow through biological tissue. - Int. J. Eng. Sci., vol.35, pp.375-385.

Received: December 21, 2013

Revised: January 15, 2015 